\title{
Spatial Clustering of Primordial Black Holes
}

\author{
Vincent Desjacques ${ }^{1}$ and A. Riotto ${ }^{2}$ \\ ${ }^{1}$ Physics department and Asher Space Science Institute, Technion, Haifa 3200003, Israel* \\ ${ }^{2}$ Département de Physique Théorique and Centre for Astroparticle Physics (CAP), \\ Université de Genève, 24 quai Ernest Ansermet, CH-1211 Geneva, Switzerland
}

(Dated: April 18, 2019)

\begin{abstract}
The possibility that primordial black holes (PBHs) are the dark matter (or a fraction thereof) has attracted much attention recently. Their spatial clustering is a fundamental property which determines, among others, whether current observational constraints are evaded within a given mass range, whether merging is significant and whether primordial black holes could generate cosmological structure. We treat them as discrete objects and clarify the issue of their spatial clustering, with an emphasis on short-range exclusion and its impact on their large scale power spectrum. Even if a Poissonian self-pair term is always present in the zero-lag correlation, this does not necessarily imply that primordial black holes are initially Poisson distributed. However, while the initial PBH clustering depends on the detailed shape of the small-scale power spectrum, we argue that it is not relevant for a narrow spectral feature and primordial black hole masses still allowed by observations.
\end{abstract}

\section{INTRODUCTION}

The physics case for the existence of Primordial Black Holes (PBHs) and for the hypothesis that all (or a fraction of) dark matter is made of them has regained momentum (see for instance Refs. [1-8] and [9] for a recent review) after the detection of gravitational waves generated by the merging of two $\sim 30 M_{\odot}$ black holes [10].

In order to robustly assess whether $\mathrm{PBHs}$ compose a significant fraction of the dark matter, one should first investigate carefully whether current observational constraints on the PBH abundances are respected in a given mass range [11]. This requires that one takes into account the possibility of $\mathrm{PBH}$ growth through rapid $\mathrm{PBH}$ merging and accretion, leading to different spatial distributions at later times [12-14]. In particular, a significant clustering of PBHs could help in avoiding microlensing constraints as well as limits from the cosmic microwave background [15] (see, however, Ref. [16] for supernovae magnification constraints). Furthermore, the merger rates of $\mathrm{PBH}$ binaries [17] along with the role played by PBHs in generating cosmological structures depend on the clustering of PBHs [18, 23]. Therefore, the spatial clustering of PBHs plays a crucial role but, surprisingly, little attention has been devoted to it.

The first detailed study of the spatial clustering of PBHs at their time of formation can be traced back to Ref. [12] (see also Ref. [19]), in which it was argued that the PBH two-point correlation function $\xi_{\mathrm{PBH}}(x)$ at smallscales is much larger than the probability to form a PBH inside a horizon volume. This led the author to conclude that the mean occupation number is much larger than unity and, thus, significantly deviates from a random, Poisson distribution. There has not been any general consensus about this conclusion (see, for instance, the

\footnotetext{
*Electronic address: dvince@physics.technion.ac.il

†Electronic address: Antonio.Riotto@unige.ch
}

footnote of section 2.2 in Ref. [9]), which has indeed been challenged very recently by Ref. [20].

It is clear that the amount of initial clustering has an impact on the subsequent evolution and thus provides an important input of the problem. The goal of this short note is not to investigate the time evolution of $\mathrm{PBH}$ clustering into the non-linear regime, a task which we believe can be performed satisfactorily only through suitable Nbody simulations, but to clarify the issue of and offer insights on their linear clustering. In particular we will show that exclusion effects have a small impact on the low- $k$ PBH power spectrum, in contrast to the findings of [12]. Furthermore, we will argue that, for reasonable parameters, the impact of their initial clustering on the formation of early PBH binaries can be neglected. This has implications as a large initial clustering makes the constraints on PBH dark matter more severe [21].

\section{GENERAL CONSIDERATIONS}

PBHs may form at a given time if the energy density perturbation is sizeable enough when the corresponding wavelengths are re-entering the horizon (after inflation). The large density contrast $\delta$ collapses to form PBHs almost immediately after horizon reentry [9] and the resulting $\mathrm{PBH}$ mass is of the order of the mass $M_{\mathrm{H}}$ contained in the corresponding horizon volume (in fact it satisfies a scaling relation with initial perturbations [22]).

We are interested in the way the PBHs are spatially distributed. To characterize the $\mathrm{PBH}$ two-point correlation function $\xi_{\mathrm{PBH}}(x)$ (or, simply, correlation function) at any comoving separation $x=|\mathbf{x}|$, we can use the the peak approach to large scale structure [24, 25]. The overdensity of discrete PBH centers at position $\mathbf{x}_{i}$ is

$$
\delta_{\mathrm{PBH}}(\mathbf{x})=\frac{1}{\bar{n}_{\mathrm{PBH}}} \sum_{i} \delta_{D}\left(\mathbf{x}-\mathbf{x}_{i}\right)-1
$$

where $\delta_{D}(\mathbf{x})$ is the three-dimensional Dirac distribution, $\bar{n}_{\mathrm{PBH}}$ is the average number density of PBH per comoving 
volume and $i$ runs over the initial positions of PBHs. The corresponding two-point correlation function must take the general form (see, for instance, Ref. [26] in the large scale structure context)

$$
\begin{aligned}
\left\langle\delta_{\mathrm{PBH}}(\mathbf{x}) \delta_{\mathrm{PBH}}(\mathbf{0})\right\rangle & =\frac{1}{\bar{n}_{\mathrm{PBH}}} \delta_{D}(\mathbf{x})-1 \\
& +\frac{1}{\bar{n}_{\mathrm{PBH}}^{2}}\left\langle\sum_{i \neq j} \delta_{D}\left(\mathbf{x}-\mathbf{x}_{i}\right) \delta_{D}\left(\mathbf{x}_{j}\right)\right\rangle \\
& =\frac{1}{\bar{n}_{\mathrm{PBH}}} \delta_{D}(\mathbf{x})+\xi_{\mathrm{PBH}}(x) .
\end{aligned}
$$

Here, $\xi_{\mathrm{PBH}}(x)$ is the reduced $\mathrm{PBH}$ correlation function. On large scales, we have

$$
\xi_{\mathrm{PBH}}(x)=b_{1}^{2} \xi_{r}(x),
$$

where $\xi_{r}(x)$ is the radiation two-point correlation function and $b_{1}$ is the linear, scale-independent PBH bias. (see [27] for a review on bias in large scale structure). We will ignore the complications of higher-order biasing throughout.

As the comoving separation $x$ decreases, the ratio $\xi_{\text {Рвн }}(x) / \xi_{r}(x)$ increases in a scale-dependent way until $x$ becomes of order the comoving size $x_{\text {exc }}$ of the smallscale exclusion volume. For most PBH scenarios, $x_{\text {exc }}$ is approximately the comoving Hubble radius $x_{\mathrm{H}}$ at formation time. This spatial exclusion arises because distinct PBHs cannot form arbitrarily close to each other. As a result, the conditional probability $p(x \mid 0)$ to find a $\mathrm{PBH}$ at a comoving distance $x$ from another $\mathrm{PBH}$, which is proportional to $1+\xi_{\mathrm{PBH}}(x)$, must vanish for $x \lesssim x_{\mathrm{exc}}$. Therefore, the reduced correlation becomes

$$
\xi_{\mathrm{PBH}}(x) \approx-1 \text { for } x \lesssim x_{\mathrm{exc}},
$$

that is, $\mathrm{PBH}$ are anti-correlated at short distances as one should expect from the fact that there is at most one $\mathrm{PBH}$ per horizon volume. This also emphasizes that, while $0 \leq p(x \mid 0) \leq 1$ surely holds, the limit $p(x \rightarrow 0 \mid 0)$ is not always unity, unlike what is assumed in Ref. [20] (see Ref. [28] for counterexamples).

Finally, at zero-lag, the "self-pairs" contribute the wellknown Poisson noise $\delta_{D}(\mathbf{x}) / \bar{n}_{\mathrm{PBH}}$. This term represents the shot noise arising from the discrete nature of the $\mathrm{PBH}$ and, thus, is present for any distribution of point-like objects regardless of their clustering.

Therefore, while Ref. [20] properly argued that the zero-lag correlation is always of the form $\delta_{D}(\mathbf{x}) / \bar{n}_{\mathrm{PBH}}$ (which is equivalent to saying that $\mathrm{PBH}$ are discrete objects), this does not automatically imply that $\mathrm{PBHs}$ are Poisson distributed at small scales. This will be the case only if shot noise fluctuations dominate over clustering effects. Because power spectra and correlation functions behave differently, we shall examine this issue in both statistics.

\section{CLUSTERING IN THE "SPIKY" $\mathrm{PBH}$ MODEL}

Let us illustrate these general considerations with an infinitely narrow spike centered at comoving wavenumber $k_{\mathrm{H}} \sim x_{\mathrm{H}}^{-1}$ on top of a smooth radiation power spectrum $P_{r}(k)$. We shall refer to this model as "spiky" PBHs. Here, $x_{\mathrm{H}} \propto M_{\mathrm{PBH}}^{1 / 2}$ is the comoving Hubble radius when the collapsing perturbation re-enters the horizon. This scaling holds in radiation domination, during which the mass enclosed by the horizon scales like $M_{\mathrm{H}} \propto \bar{\rho}_{r} H^{-3} \propto$ $H^{-1}$ while the comoving Hubble radius is given by $x_{\mathrm{H}}=$ $(a H)^{-1} \propto H^{-1 / 2}$. In particular, $k_{\mathrm{H}} \sim 4 \times 10^{6} \mathrm{Mpc}^{-1}$ for $\mathrm{PBH}$ of mass $M_{\mathrm{PBH}} \sim M_{\odot}$. Therefore,

$$
P_{r}(k)=P_{l}(k)+\frac{2 \pi^{2} \sigma_{s}^{2}}{k^{2}} \delta_{D}\left(k-k_{\mathrm{H}}\right),
$$

where $P_{l}(k)$ is the power spectrum of the long-wavelength (adiabatic) fluctuations $\delta_{l}$ in the radiation density field, while $\sigma_{s}$ is the rms variance of the short-wavelength fluctuations $\delta_{s}$ that collapse to form PBHs. The latter reenter the horizon very early. The former imprint longwavelength fluctuations in the number density of $\mathrm{PBH}$ and, therefore, generates a bias analogous to the peakbackground split bias of dark matter halos [12].

\section{A. Linear scales}

On scales $k \ll k_{\mathrm{H}}$, the $\mathrm{PBH}$ bias is surely linear and scale-independent for the Gaussian initial conditions assumed here. In the high peak limit (see, e.g., [24]), it is given by

$$
b_{1} \sim \frac{\delta_{c}}{\sigma_{0}^{2}\left(x_{\mathrm{H}}\right)} \sim \frac{\delta_{c}}{\sigma_{s}^{2}} \equiv \frac{\nu_{s}}{\sigma_{s}},
$$

where $\nu_{s}$ is the peak significance and $\delta_{c}$ is the critical overdensity that leads to black hole formation. Furthermore, $\sigma_{n}^{2}\left(x_{\mathrm{H}}\right)=\sigma_{n l}^{2}\left(x_{\mathrm{H}}\right)+\sigma_{s}^{2} k_{\mathrm{H}}^{2 n}$ are the spectral moments $\left\langle k^{2 n}\right\rangle$ of the radiation power spectrum smoothed on comoving scale $x_{\mathrm{H}}$ :

$$
\sigma_{n}^{2}(R)=\frac{1}{2 \pi^{2}} \int_{0}^{\infty} d k k^{2} P_{r}(k) W^{2}(k R),
$$

where $W(k R)$ is the Fourier transform of the spherically symmetric window function (assumed to be a tophat throughout). The second approximation in Eq.(6) follows from the fact that $\sigma_{s}^{2} k_{\mathrm{H}}^{2 n}$ is typically much larger than the spectral moment $\sigma_{n l}^{2}\left(x_{\mathrm{H}}\right)$ produced by the longwavelength piece of the radiation field solely. Therefore, the linear PBH bias (relative to the radiation distribution) will be significantly larger than unity as soon as the variance $\sigma_{s}^{2}$ is less than a few $\times 0.1$.

We do not consider the case in which a $\mathrm{PBH}$ can be contained into a bigger PBH. In large scale structure, this is known as the cloud-in-cloud problem. While this is not 
an issue for the monochromatic spike discussed here, and the narrow feature considered later, it must be taken into account when considering a broad feature.

At small scales, $\mathrm{PBH}$ s exclude each other because of the finite size $x_{\mathrm{H}}$ of the horizons that collapse to form PBHs, as discussed above. Upon a Fourier transform, this localized effect in configuration space affects the $\mathrm{PBH}$ power spectrum $P_{\mathrm{PBH}}(k)$ at small $k$. Since the behavior of $P_{\mathrm{PBH}}(k)$ in the limit $k \rightarrow 0$ is central to the argument of [12] about the clustering of $\mathrm{PBHs}$, we shall revisit it now.

\section{B. PBH shot noise at low wavenumber}

The clustering of PBHs imply that the low- $k$ white noise contribution to $P_{\mathrm{PBH}}(k)$ deviates from the naive Poisson expectation $1 / \bar{n}_{\mathrm{PBH}}$. This effect has recently been studied in the context of halo clustering [26, 28-30, 32]. More precisely, at small wavenumbers the $\mathrm{PBH}$ power spectrum is

$$
P_{\mathrm{PBH}}(k) \stackrel{k \rightarrow 0}{=} \frac{1}{\bar{n}_{\mathrm{PBH}}}+\int \mathrm{d}^{3} x \xi_{\mathrm{PBH}}(x),
$$

which follows from the Fourier transform of Eq. (2). Note that this result is fully general and, thus, applies to any PBH model.

The volume integral over $\xi_{\mathrm{PBH}}(x)$ is constrained to be larger than $-1 / \bar{n}_{\mathrm{PBH}}$, so that $P_{\mathrm{PBH}}(k)$ is always positive definite. Furthermore, it depends sensitively on the details of the $\mathrm{PBH}$ clustering. In fact, it can be positive or negative and, thus, leads to super- or subPoissonian noise in the low- $k$ power spectrum, respectively. Ref. [12] computed the integral over $\xi_{\mathrm{PBH}}(x)$ for "spiky" PBHs using the "highly biased regions" approximation of Ref. [31] and, moreover, truncated the integral at the lower cutoff $x=x_{\mathrm{H}}$ (rather than $x=0$ ), finding $P_{\mathrm{PBH}}(k \rightarrow 0)>1 / \bar{n}_{\mathrm{PBH}}$. However, extending the integral down to $x=0$ might have reversed the sign of this integrated contribution if small-scale exclusion is significant. This effect will not be present unless a peak constraint [25] is enforced, 1.e. if one requires the radiation overdensity $\delta_{r}$ to reach a local maximum at the $\mathrm{PBH}$ position. Moreover, Ref. [12] extrapolated the condition $\xi_{\mathrm{PBH}}(r) / \xi_{\mathrm{PBH}}(0) \gg 1 / \nu_{s}$ in regimes where it is not valid [20].

\section{C. "Halo model" estimate of the PBH shot noise}

Although peak theory has built-in spatial exclusion, calculations are not easily tractable in this framework because the effect is highly non-perturbative [see, e.g., 26, 28]. To estimate the deviation from Poisson noise in the low- $k \mathrm{PBH}$ power spectrum, we shall therefore proceed along the lines advocated by Refs. [29, 32] and attempt to establish a correspondence with the halo model approach to large scale structure. In this framework, all dark matter is bound to halos of a wide range of mass $M$. Consider now a narrow mass range centered at $M_{i}$. At small wavenumbers, the Fourier modes $\delta_{i}(\mathbf{k})$ of the resulting halo fluctuation field take the general form

$$
\delta_{i}(\mathbf{k})=b_{i} \delta_{m}(\mathbf{k})+\epsilon_{i}(\mathbf{k})
$$

where $\epsilon_{i}(\mathbf{k})$ is the shot noise contribution. Massmomentum conservation implies that the mass-weighted sum of the shot noise power spectra, $\sim \sum_{i} M_{i}^{2} P_{\epsilon_{i}}(k)$, converges towards zero in the limit $k \rightarrow 0$. As shown in Ref. [29, 32], this condition can be used to obtain a consistent prediction for $P_{\epsilon_{i}}(k)$ from the halo model. In plain words, one finds the following scaling

$$
P_{\epsilon_{i}}(k \rightarrow 0) \sim \frac{1}{\bar{n}_{i}}\left(1-b_{1} \bar{n}_{i} V_{\text {exc }}\right)^{2},
$$

where the (comoving) exclusion volume $V_{\text {exc }} \equiv M_{i} / \bar{\rho}_{m}$ is exactly given by the Lagrangian volume of the halos. This relation includes also the effect of the large scale clustering through the factor $b_{1}$.

In order to apply these considerations to PBHs, we draw the correspondence

$$
\begin{aligned}
\text { halos } & \Leftrightarrow \quad \mathrm{PBHs} \\
\text { matter } & \Leftrightarrow \quad \text { radiation, }
\end{aligned}
$$

and take the radiation perturbation $\delta_{r}$ to be in synchronous gauge (comoving with the nonrelativistic matter). This is the equivalent of the Lagrangian space used for dark matter halos [33, 34]. Although a fraction only of the radiation component indeed collapses to form $\mathrm{PBHs}$, the similarity with the halo model calculation is still valid. Therefore, on large scales, the PBH overabundance is described by

$$
\delta_{\mathrm{PBH}}(\mathbf{k})=b_{1} \delta_{r}(\mathbf{k})+\epsilon(\mathbf{k})
$$

where $b_{1}$ is now defined relative to $\delta_{r}(\mathbf{k})$ instead of $\delta_{m}(\mathbf{k})$. Furthermore, the shot noise contribution $P_{\epsilon}(k)$ is given by Eq. (10), provided that $\bar{n}_{i}$ is replaced by $\bar{n}_{\mathrm{PBH}}$ and the characteristic volume is given by $V_{\text {exc }}=M_{\mathrm{PBH}} / \bar{\rho}_{r}=$ $(4 \pi / 3) x_{\mathrm{H}}^{3}$. Therefore, $\mathrm{PBH}$ exclusion effects on the small $k$ power spectrum will be significant when

$$
b_{1} x_{\mathrm{H}}^{3} \gtrsim \bar{x}^{3}
$$

Here, $\bar{x}=\left(3 / 4 \pi \bar{n}_{\mathrm{PBH}}\right)^{1 / 3}$ is the average comoving separation between PBHs. For the monochromatic spectrum considered here, this condition implies that the PBH linear bias be

$$
b_{1} \gtrsim a_{\mathrm{H}}^{-1}\left(\frac{\Omega_{r}}{\Omega_{\mathrm{PBH}}}\right) \text {, }
$$

where $a_{\mathrm{H}}$ is the scale factor at horizon entry, while $\Omega_{r}$ and $\Omega_{\mathrm{PBH}}$ are the present-day radiation and $\mathrm{PBH}$ energy densities. If $\mathrm{PBHs}$ make all the dark matter, 
Eq. (14) reduces to (assuming a number of relativistic d.o.f. $\left.g_{*}\left(a_{\text {eq }}\right)=3.36\right)$

$$
b_{1} \gtrsim\left(\frac{a_{\mathrm{eq}}}{a_{\mathrm{H}}}\right) \simeq 2.9 \times 10^{9}\left(\frac{M_{\mathrm{PBH}}}{M_{\odot}}\right)^{-1 / 2},
$$

where $a_{\mathrm{eq}}$ is the scale factor at matter-radiation equality.

We emphasize that Eq.(15) is a criterion for the importance of a non-Poissonian correction to the low- $k \mathrm{PBH}$ power spectrum. The importance of the $\mathrm{PBH}$ correlation function, which is relevant to the formation of early $\mathrm{PBH}$ binaries, will be discussed below.

Clearly, the correction to Poisson shot noise in the power spectrum is completely negligible unless $\mathrm{PBH}$ s are simultaneously very massive (but $M_{\mathrm{PBH}} \gtrsim 10^{3} M_{\odot}$ is severely constrained by the data [9]) and strongly clustered with $b_{1} \gg 1$. If all dark matter is PBHs, the white noise power induced by the PBH clustering and discreteness can thus be taken as Poissonian, in contrast to the finding of [12].

We stress, however, that this is not incompatible with the fact that they can significantly cluster on sufficiently small scales. For the formation of $\mathrm{PBH}$ binaries in particular, what truly matters are the moments of the counts of neighbors (see [35] for a review). In particular, the mean count $\langle N\rangle$ in a cell of volume $V$ centered on a $\mathrm{PBH}$ is

$$
\langle N\rangle=\bar{n}_{\mathrm{PBH}} V+\bar{n}_{\mathrm{PBH}} \int_{V} \mathrm{~d}^{3} x \xi_{\mathrm{PBH}}(x) .
$$

$\langle N\rangle$ significantly deviates from Poisson if the contribution $\bar{n}_{\mathrm{PBH}} \int_{V} \mathrm{~d}^{3} x \xi_{\mathrm{PBH}}(x)$ from $\mathrm{PBH}$ clustering is larger than the discreteness noise $\bar{n}_{\mathrm{PBH}} V$. To quantify the importance of the former, we will compute the characteristic (comoving) clustering length $x_{\xi}(a)$ defined through the relation $\xi_{\mathrm{PBH}}\left(x_{\xi}(a), a\right)=1$. Since the clustering is hierarchical for realistic initial power spectra, the second term in the right-hand side of Eq.(16) becomes larger than the first when the cell radius is $\lesssim x_{\xi}(a)$.

\section{INITIAL CLUSTERING AND FORMATION OF PBH BINARIES}

For the formation of $\mathrm{PBH}$ binaries in the early Universe [39], the initial $\mathrm{PBH}$ clustering is the key relevant quantity $[40,41]$. The first question is whether one should compute it at horizon crossing $a=a_{*}$, or at the formation epoch $a=a_{\mathrm{H}}$ of $\mathrm{PBH}$. Here, $a_{*}$ defined through $k=a_{*} H\left(a_{*}\right)$ is the scale factor at which a perturbation of wavenumber $k$ crosses the horizon.

\section{A. The initial $\mathrm{PBH}$ power spectrum}

We argue that the two alternatives must return the same answer because no physical process can affect the relative separation $x$ between two PBHs so long as $x$ is larger than the horizon. This can be captured through the requirement that $\delta_{\mathrm{PBH}}(\mathbf{k}, a) \approx b_{1}(a) \delta_{r}(\mathbf{k}, a)$ remains constant for $a<a_{*}$,

$$
a^{2} b_{1}(a)=\mathrm{const} \quad \text { for } \quad a<a_{*} .
$$

More precisely, consider the evolution of a wavemode $\delta_{r}(\mathbf{k}, a)$ with $k<k_{\mathrm{H}}$ until it crosses the horizon at $a_{\mathrm{H}}<a_{*}<a_{\mathrm{eq}}$. Since $k=a_{*} H\left(a_{*}\right)$ and we are in radiation domination, $a_{*} / a_{\mathrm{eq}}=k_{\mathrm{eq}} / k$. The amplitude of this mode at $a=a_{*}$ is given by

$$
\begin{aligned}
\delta_{r}\left(\mathbf{k}, a_{*}\right) & =\delta_{r}\left(\mathbf{k}, a_{\mathrm{H}}\right)\left(\frac{a_{*}}{a_{\mathrm{H}}}\right)^{2} \\
& =\delta_{r}\left(\mathbf{k}, a_{\mathrm{H}}\right)\left(\frac{a_{\mathrm{eq}}}{a_{\mathrm{H}}}\right)^{2}\left(\frac{k_{\mathrm{eq}}}{k}\right)^{2} .
\end{aligned}
$$

where $k_{\text {eq }}^{2} \equiv 2 \Omega_{m} H_{0}^{2} / a_{\text {eq }}$ defines the comoving wavenumber corresponding to matter-radiation equality. Since the radiation power spectrum at horizon crossing is

$$
P_{r}\left(k, a_{*}\right)=\left(\frac{4}{9}\right)^{2} P_{\zeta}(k),
$$

the radiation power spectrum at black hole formation reads

$$
P_{r}\left(k, a_{\mathrm{H}}\right)=\left(\frac{4}{9}\right)^{2}\left(\frac{a_{\mathrm{H}}}{a_{\mathrm{eq}}}\right)^{4}\left(\frac{k}{k_{\mathrm{eq}}}\right)^{4} P_{\zeta}(k) .
$$

Using Eq.(17), we immediately see that

$$
b_{1}^{2}\left(a_{\mathrm{H}}\right) P_{r}\left(k, a_{\mathrm{H}}\right)=b_{1}^{2}\left(a_{*}\right) P_{r}\left(k, a_{*}\right),
$$

i.e. the two alternatives consistently give the same answer. Note that these power spectra are computed before the transfer function epoch (The Meszaros effect [2] is thus not included).

To estimate the initial $\mathrm{PBH}$ correlation $\xi_{\mathrm{PBH}}$, it is convenient to start from $P_{\mathrm{PBH}}\left(k, a_{\mathrm{H}}\right)=b_{1}^{2}\left(a_{\mathrm{H}}\right) P_{r}\left(k, a_{\mathrm{H}}\right)$ since $\mathrm{PBH}$ formation models naturally furnish an estimate of $b_{1}\left(a_{\mathrm{H}}\right)$. Namely, for a peak significance $\nu_{s}=\mathcal{O}(10)$ corresponding to a PBH mass fraction of the order of $10^{-9}\left(M_{\mathrm{PBH}} / M_{\odot}\right)^{1 / 2}$, and a rms variance $\sigma_{s} \sim 0.1$ on the $\mathrm{PBH}$ scale, the linear bias at $\mathrm{PBH}$ formation is of order $b_{1}\left(a_{\mathrm{H}}\right) \sim \mathcal{O}\left(10^{2}\right)$ upon applying the high peak result Eq. (6).

For the "spiky" PBH model Eq.(5), with $P_{l}\left(k, a_{\mathrm{H}}\right)$ given by Eq.(20), the initial $\mathrm{PBH}$ power spectrum reads

$$
\begin{aligned}
P_{\mathrm{PBH}}\left(k, a_{\mathrm{H}}\right) & =b_{1}^{2}\left(a_{\mathrm{H}}\right)\left[P_{l}\left(k, a_{\mathrm{H}}\right)+\frac{2 \pi^{2} \sigma_{*}^{2}}{k^{2}} \delta_{D}\left(k-k_{\mathrm{H}}\right)\right] \\
\sim 10^{4} & \left(\frac{b_{1}\left(a_{\mathrm{H}}\right)}{10^{2}}\right)^{2}\left[10^{-39}\left(\frac{M_{\mathrm{PBH}}}{M_{\odot}}\right)^{2}\left(\frac{k}{k_{\mathrm{eq}}}\right)^{4} P_{\zeta_{l}}(k)\right. \\
& \left.+\frac{2 \pi^{2} \sigma_{*}^{2}}{k^{2}} \delta_{D}\left(k-k_{\mathrm{H}}\right)\right]
\end{aligned}
$$

where $P_{\zeta_{l}}(k)$ is the smooth component of the primordial curvature power spectrum. The smallness of the 
numerical factor in the contribution from $P_{l}\left(k, a_{\mathrm{H}}\right)$ arises from $\left(a_{\mathrm{H}} / a_{\mathrm{eq}}\right)^{4}$. Furthermore, we have replaced $\sigma_{s}\left(a_{\mathrm{H}}\right)$ in Eq.(5) by a free rms variance $\sigma_{*}$. The reason for this choice will made be clear below.

We can now compute $\xi_{\mathrm{PBH}}\left(x, a_{\mathrm{H}}\right)$ by Fourier transforming $P_{\mathrm{PBH}}\left(k, a_{\mathrm{H}}\right)$.

\section{B. Clustering length}

The PBH correlation function can be expressed as

$$
\xi_{\mathrm{PBH}}\left(x, a_{\mathrm{H}}\right)=\frac{1}{2 \pi^{2}} \int_{0}^{\infty} d k k^{2} P_{\mathrm{PBH}}\left(k, a_{\mathrm{H}}\right) j_{0}(k x)
$$

For a scale-invariant primordial curvature power spectrum

$$
\frac{k^{3}}{2 \pi^{2}} P_{\zeta_{l}}(k)=A_{s} \quad \text { with } \quad A_{s} \sim 10^{-9},
$$

the contribution from the smooth, long-wavelength piece $P_{l}\left(k, a_{\mathrm{H}}\right)$ of the radiation power spectrum is (momentarily omitting the multiplicative factor of $b_{1}^{2}$ to avoid clutter)

$$
\begin{gathered}
\frac{10^{-35}}{2 \pi^{2}}\left(\frac{M_{\mathrm{PBH}}}{M_{\odot}}\right)^{2} \int_{0}^{\infty} d k k^{2}\left(\frac{k}{k_{\mathrm{eq}}}\right)^{4} P_{\zeta_{l}}(k) j_{0}(k x) \\
=10^{-35} A_{s}\left(\frac{M_{\mathrm{PBH}}}{M_{\odot}}\right)^{2} \int_{0}^{\infty} d k \frac{k^{3}}{k_{\mathrm{eq}}^{4}} j_{0}(k x) \\
\simeq 10^{-35} A_{s}\left(\frac{M_{\mathrm{PBH}}}{M_{\odot}}\right)^{2}\left(k_{\mathrm{eq}} x\right)^{-4} .
\end{gathered}
$$

To obtain the last equality, we have taken advantage of the fact that the spherical bessel function satisfies $j_{0}(k x) \approx 1$ for $k \lesssim 1 / x$, and quickly drops to zero for $k \gtrsim 1 / x$.

The calculation of the contribution to $\xi_{\mathrm{PBH}}\left(x, a_{\mathrm{H}}\right)$ arising from the spike proceeds analogously. We find

$$
\begin{aligned}
& \frac{10^{4}}{2 \pi^{2}} \int_{0}^{\infty} d k k^{2}\left(\frac{2 \pi^{2} \sigma_{*}^{2}}{k^{2}} \delta_{D}\left(k-k_{\mathrm{H}}\right)\right) j_{0}(k x) \\
& \quad \simeq 10^{2}\left(\frac{\sigma_{*}}{0.1}\right)^{2} j_{0}\left(k_{\mathrm{H}} x\right) .
\end{aligned}
$$

The PBH correlation function is the sum of Eq.(25) and Eq.(26).

To estimate the relative amplitude of these two terms, we note that, in the limit $x \rightarrow \infty$, the contribution arising from the smooth power spectrum $P_{l}\left(k, a_{\mathrm{H}}\right)$ decays as $x^{-4}$ while that arising from the spike asymptotes to a constant. Therefore, the latter clearly dominates at large scales. Furthermore, $x$ cannot be taken smaller than $\sim 1 / k_{\mathrm{H}}$. The reason is that fluctuations of wavenumber $k \gtrsim k_{\mathrm{H}}$ cannot increase the initial clustering of $\mathrm{PBH}$ centers because their wavelength is smaller than the size of the patches which collapse to form $\mathrm{PBH}$. In fact, those scales will contribute to decrease the amplitude of clustering through the exclusion effect discussed above. Therefore, the contribution from $P_{l}\left(k, a_{\mathrm{H}}\right)$ does not exceed the upper limit (we have now restored the factor of $b_{1}^{2}\left(a_{\mathrm{H}}\right)$ )

$$
\begin{gathered}
10^{-35} A_{s}\left(\frac{b_{1}\left(a_{\mathrm{H}}\right)}{10^{2}}\right)^{2}\left(\frac{M_{\mathrm{PBH}}}{M_{\odot}}\right)^{2}\left(\frac{k_{\mathrm{H}}}{k_{\mathrm{eq}}}\right)^{4} \\
\simeq 10^{4} A_{s}\left(\frac{b_{1}\left(a_{\mathrm{H}}\right)}{10^{2}}\right)^{2}
\end{gathered}
$$

where we have used the fact that $a_{\mathrm{H}} k_{\mathrm{H}}=a_{\mathrm{eq}} k_{\mathrm{eq}}$. Since $A_{s} \sim 10^{-9}$, this upper limit is still much smaller than the contribution Eq.(26) arising from the narrow feature unless the linear bias is unrealistically large, $b_{1}\left(a_{\mathrm{H}}\right) \gg$ $10^{2}$. Therefore, the dominant contribution to the initial $\mathrm{PBH}$ clustering arises from the spike itself.

We can now estimate the initial PBH clustering length $x_{\xi}\left(a_{\mathrm{H}}\right)$ upon demanding that $\xi_{\mathrm{PBH}}\left(x_{\xi}\left(a_{\mathrm{H}}\right), a_{\mathrm{H}}\right) \sim 1$, where $\xi_{\text {РBH }}$ is given by Eq.(26). Since we want the clustering length to be significantly larger than the horizon size $x_{\mathrm{H}}$, the argument of $j_{0}$ is $k_{\mathrm{H}} x_{\xi} \gg 1$. Therefore, we can approximate the spherical bessel function by its envelop, $j_{0}\left(k_{\mathrm{H}} x_{\xi}\right) \sim\left(k_{\mathrm{H}} x_{\xi}\right)^{-1}$, to find

$x_{\xi}\left(a_{\mathrm{H}}\right) \simeq 3 \times 10^{-5}\left(\frac{\sigma_{*}}{0.1}\right)^{2}\left(\frac{b_{1}\left(a_{\mathrm{H}}\right)}{10^{2}}\right)^{2}\left(\frac{M_{\mathrm{PBH}}}{M_{\odot}}\right)^{1 / 2} \mathrm{Mpc}$

where, again, we have restored the multiplicative bias factor for the sake of the following discussion.

\section{Implications}

The results are sensitive to the choice of $\sigma_{*}$. Adopting $\sigma_{*} \equiv \sigma_{s}\left(a_{\mathrm{H}}\right)$ implies that we include the high density fluctuations that collapse to form $\mathrm{PBH}$ in the calculation of the clustering of $\mathrm{PBH}$ centers. This does not seem to make sense. In the halo model approach to large scale structure, it would be like computing the clustering of halo centers including the 1-halo term. Therefore, these large fluctuations should be excised from the calculation, suggesting that the effective $\sigma_{*}$ is significantly smaller than $\sigma_{s}\left(a_{\mathrm{H}}\right) \sim 0.1$.

For the interesting case of $\mathrm{PBHs}$ with $M_{\mathrm{PBH}} \sim 30 M_{\odot}$ (now severely constrained by the data if they are all the dark matter), the range of initial distances relevant for the calculation of the present merger rate is $\gtrsim 4 \cdot 10^{-5}$ Mpc [43]. Our estimate Eq.(28) indicates that $x_{\xi}\left(a_{\mathrm{H}}\right)$ can reach such values only if $\sigma_{*}$ is close to $\sigma_{s}\left(a_{\mathrm{H}}\right)=0.1$. For $\sigma_{*} \ll \sigma_{s}\left(a_{\mathrm{H}}\right)$ which, as we argued above, likely reflects the contribution of the spike to the clustering of $\mathrm{PBH}$ centers, $x_{\xi}$ would be much smaller than $10^{-5} \mathrm{Mpc}$. Therefore, since for $\sigma_{*}=\sigma_{s}\left(a_{\mathrm{H}}\right), x_{\xi}$ does not exceed significantly the bound $\gtrsim 4 \cdot 10^{-5} \mathrm{Mpc}$ found by [43], we conclude that the initial clustering is not relevant for solar mass PBHs if they collapse out of a narrow feature in the primordial curvature power spectrum. 
It is also instructive to express $x_{\xi}$ in unit of the mean comoving $\mathrm{PBH}$ separation $\bar{x}$. Assuming $\Omega_{\mathrm{PBH}} \sim \mathcal{O}(0.1)$ as adopted throughout our calculation, we find

$$
\frac{x_{\xi}\left(a_{\mathrm{H}}\right)}{\bar{x}} \simeq 0.1\left(\frac{\sigma_{*}}{0.1}\right)^{2}\left(\frac{b_{1}\left(a_{\mathrm{H}}\right)}{10^{2}}\right)^{2}\left(\frac{M_{\mathrm{PBH}}}{M_{\odot}}\right)^{1 / 6}
$$

This shows that, for a mass $M_{\mathrm{PBH}} \sim 30 M_{\odot}$, the condition $x_{\xi}\left(a_{\mathrm{H}}\right) \sim \bar{x}$ can be marginally achieved only if $\sigma_{*} \sim \sigma_{s}\left(a_{\mathrm{H}}\right)$. Hence, our findings show that, even if the initial distribution of $\mathrm{PBHs}$ at formation time can potentially differ from a Poissonian, this does not happen for solar mass PBHs and for the narrow feature considered here. For the small mass window $M_{\mathrm{PBH}} \sim 10^{-12} M_{\odot}$, we find $x_{\xi} \ll \bar{x}$ which shows that, here again, the initial clustering is not significant.

We stress that the conclusions presented here do not apply to broad features. In this case, one should discard small PBHs that are quickly swallowed by bigger PBHs, and keep only the later. This cloud-in-cloud problem could be addressed using, e.g., the excursion set approach pioneered in [42], but this is beyond the scope of this paper.

\section{CONCLUSIONS}

In this paper, we have discussed the basic features of $\mathrm{PBH}$ spatial clustering treating them as discrete objects. We have delineated the relation between the large- distance $\mathrm{PBH}$ power spectrum and short-range $\mathrm{PBH}$ exclusion effects. When all dark matter is in the form of PBHs, the white noise can be taken as Poissonian, in contrast to the findings of [12]. We have also emphasized that, while the zero-lag correlation includes a Poissonian self-pair contribution, this does not mean that PBHs are necessarily Poisson distributed at small scales. Therefore, we also disagree with some of the arguments presented in [20]. Hierarchical clustering implies that clusteringinduced fluctuations dominate on scales less than the characteristic PBH clustering length, while Poisson fluctuations dominate on large scales. While the exact value of the initial clustering length is sensitive to the shape of the primordial curvature power spectrum, our estimates suggest that, for a narrow feature, the characteristic $\mathrm{PBH}$ clustering length is significantly smaller than the mean comoving PBH separation for resonable set of the parameters, rendering clustering not relevant.

\section{Acknowledgments}

We thank Y. Ali-Haimoud for comments on an earlier version of this manuscript, and Chris Byrnes for a stimulating correspondence. V.D. is grateful to Ely Kovetz and Alvise Raccanelli for helpful discussions, and acknowledges support by the Israel Science Foundation (grant no. 1395/16). A.R. is supported by the Swiss National Science Foundation (SNSF), project Investigating the Nature of Dark Matter, project number: 200020-159223.
[1] B. J. Carr and S. W. Hawking, Mon. Not. Roy. Astron. Soc. 168, 399 (1974).

[2] P. Meszaros, Astron. Astrophys. 37, 225 (1974).

[3] B. J. Carr, Astrophys. J. 201, 1 (1975).

[4] S. Bird, I. Cholis, J. B. Munoz, Y. Ali-Haimoud, M. Kamionkowski, E. D. Kovetz, A. Raccanelli and A. G. Riess, Phys. Rev. Lett. 116, no. 20, 201301 (2016) [astro-ph.C0/1603.00464].

[5] A. Kashlinksy, Astrophys. J. Lett. 823, L25 (2016) [astro-ph.C0/1605.04023]

[6] S. Clesse and J. Garcia-Bellido, Phys. Dark Univ. 15, 142 (2017) [astro-ph.CO/1603.05234]

[7] M. Sasaki, T. Suyama, T. Tanaka and S. Yokoyama, Phys. Rev. Lett. 117, no. 6, 061101 (2016) [astro-ph.C0/1603.08338].

[8] B. Carr, M. Raidal, T. Tenkanen, V. Vaskonen and H. Veerm, Phys. Rev. D 96, no. 2, 023514 (2017) [astro-ph.C0/1705.05567].

[9] M. Sasaki, T. Suyama, T. Tanaka and S. Yokoyama, [astro-ph.Co/1801.05235].

[10] B. P. Abbott et al. [LIGO Scientific and Virgo Collaborations], Phys. Rev. Lett. 116, 061102 (2016) [gr-qc/1602.03837]

[11] B.B. Carr, F. Kuhnel, and M. Sandstad, Phys. Rev. D 94, 083504 (2016), [astro-ph.CO/1607.06077].

[12] J. R. Chisholm, Phys. Rev. D 73, 083504 (2006) [astro-ph/0509141].
[13] J. R. Chisholm, Phys. Rev. D 84, 124031 (2011) [astro-ph.c0/1110.4402].

[14] J. García-Bellido, J. Phys. Conf. Ser. 840, no. 1, 012032 (2017) [astro-ph.C0/1702.08275].

[15] J. García-Bellido and S. Clesse, Phys. Dark Univ. 19, 144 (2018) [astro-ph.CO/1710.04694].

[16] M. Zumalacarregui and U. Seljak, [astro-ph.Co/1712.02240].

[17] S. Clesse and J. Garc-Bellido, [astro-ph.CO/1711.10458].

[18] B. Carr and J. Silk, [astro-ph.Co/1801.00672] .

[19] Y. Tada and S. Yokoyama, Phys. Rev. D 91, no. 12, 123534 (2015) [astro-ph.CO/1502.01124].

[20] Y. Ali-Haoud, [astro-ph.C0/1805.05912].

[21] T. Bringmann, P. F. Depta, V. Domcke and K. SchmidtHoberg, [astro-ph.Co/1808.05910].

[22] K. Jedamzik and J. C. Niemeyer, Phys. Rev. D 59 (1999), 124014 [astro-ph/9901293].

[23] M. Raidal, V. Vaskonen and H. Vermae, JCAP 09, 037 (2017) [astro-ph.CO/1070.01480]

[24] N. Kaiser, Astrophys. J. Lett., 284, L9 (1984)

[25] J. M. Bardeen, J. R. Bond, N. Kaiser, A. S. Szalay, Astrophys. J., 304, 15 (1986)

[26] T. Baldauf, U. Seljak, R. E. Smith, N. Hamaus, V. Desjacques, Phys. Rev. D, 88, 083507 (2013) [astro-ph.CO/1305.2917].

[27] V. Desjacques, D. Jeong, F. Schmidt, Phys. Rep., 733, 1 
- 193 (2018).

[28] T. Baldauf, S. Codis, V. Desjacques, C. Pichon, Mon. Not. R. Astron. Soc., 456, 3985 (2016) [astro-ph.CO/1510.09204].

[29] N. Hamaus, U. Seljak, V. Desjacques, R. E. Smith, T. Baldauf, Phys. Rev. D, 82, 043515 (2010) [astro-ph.CO/1004.5377].

[30] F. Schmidt, Phys. Rev. D, 93, 063512 (2016) [astro-ph.C0/1511.02231].

[31] L. G. Jensen, A. S. Szalay, Astrophys. J. Lett., 305, L5 (1986)

[32] D. Ginzburg, V. Desjacques, K. C. Chan, Phys. Rev. D, 96, 083528 (2017) [astro-ph.C0/1706.08738]

[33] J. C. Hwang, H. Noh, Phys. Rev. D, 73, 044021 (2006) [astro-ph/0601041]

[34] D. Wands, A. Slosar, Phys. Rev. D, 79, 123507 (2009) [astro-ph.CO/0902.1084]

[35] P. J. E. Peebles, "The large-scale structure of the uni- verse", Princeton University Press (1980)

[36] M. Davis, P. J. E. Peebles, Astrophys. J., 267, 465 (1983)

[37] J. Loveday, S. J. Maddox, G. Efstathiou, B. A. Peterson, Astrophys. J., 442, 457 (1995) [astro-ph/9410018]

[38] S. D. M. White, in "Cosmology and Large Scale Structure", 349 (1996)

[39] T. Nakamura, M. Sasaki, T. Tanake, K. S. Thorne, Astrophys. J. Lett.. 487, L139 (1997) [astro-ph/9708060]

[40] K. Ioka, T. Chiba, T. Tanaka, T. Nakamura, Phys. Rev. D, 58, 063003 (1998) [astro-ph/9807018]

[41] Y. Ali-Haimoud, E. D. Kovetz, M. Kamionkowski, Phys. Rev. D, 96, 123523 (2017) [astro-ph.Co/1709.06576]

[42] J. R. Bond, S. Cole, G. Efstathiou, N. Kaiser, Astrophys. J., 379, 440 (1991)

[43] G. Ballesteros, P. D. Serpico, M. Taoso, [astro-ph.C0/1807.02084].

[44] C. Germani and I. Musco, [astro-ph.Co/1805.04087]. 